Oikos 121: 1665-1679, 2012

doi: $10.1111 / j .1600-0706.2011 .20259 . x$

(C) 2012 The Authors. Oikos (C) 2012 Nordic Society Oikos

Subject Editor: Justin Travis. Accepted 1 November 2011

\title{
Host-parasitoid persistence over variable spatio-temporally susceptible habitats: bottom-up effects of ephemeral resources
}

\author{
Carolina Reigada and Marcus A. M. de Aguiar \\ C. Reigada (ca.reigada@gmail.com) and M. A. M. de Aguiar, Instituto de Fisica Gleb Wataghin, Univ. Estadual de Campinas, Unicamp \\ 13083-970, Campinas, SP, Brazil.
}

\begin{abstract}
We experimentally and theoretically investigated the persistence of hosts and parasitoids interacting in a metapopulation structure consisting of ephemeral local patches (MELPs). We used a host-parasitoid system consisting of necrophagous Diptera species and their pupal parasitoids. The basal resources used by the host species were assumed to be ephemeral, supporting only one generation of individuals before completely disappearing from the environment. We experimentally measured the host-parasitoid persistence and the effects of local demographic processes in two scenarios: 1) constant occurrence of basal resources at a single site (no dispersion or colonization of other sites) and 2) variable occurrence of basal resources between two sites (colonization of a new patch requiring species dispersal). The experimental setup and findings were then formalized into a mathematical model describing the interaction dynamics in a MELP structure. We evaluated the contribution of several factors to the host-parasitoid coexistence, such as resource allocation probability (probability of resource appearance in a site), variation in resource size and number of sites available to receive resources in the MELP. We found that demographic fluctuations and environmental stochasticity affected the density of migrants, patch habitat connectivity, persistence and spatial distribution of interacting species.
\end{abstract}

In nature, it is very common that patchiness in the distribution of resources causes aggregation of consumer individuals in restricted areas of a landscape. Therefore, the inclusion of spatial structure in ecological models has become an important issue for understanding the species distribution patterns in an environment (Hassell et al. 1991, Hanski 1999). Moreover, local populations may not persist without a metapopulation structure, in which the balance between extinction and colonization of local populations, linked by dispersal, ensures species persistence at the global level (Levins 1969, Harrison 1991, Hanski 1999).

Classical metapopulation studies focus on the role of local population extinctions and the subsequent recolonization by dispersers after a few generations (Hanski 1999, Harrison 1991). However, direct recolonization may not be possible in some contexts (Harrison 1991). Examples of this situation can be observed in aggregations of organisms that breed in microhabitats, such as mushrooms, carrion, decaying wood and rock pools (Murphy et al. 1990, Harrison 1991, Hanski 1999, Altermatt and Ebert 2010). These types of microhabitats are ephemeral basal resources because they support only one or a few generations of individuals before completely disappearing from the environment. After the resources are gone, recolonization becomes impossible.

In metapopulations consisting of ephemeral local patches (termed MELP in this study), the extinction of a local population depends on the lifetime of the resources.
In this paper, we use the word patch for a spatial location (or site) where ephemeral resources are available. A site receives basal resources and turns into a patch (an ephemeral habitat to breed species) on a random basis, rather than undergoing constant replenishment of resources with every generation. When extinction occurs in an ephemeral patch, the corresponding site is frequently not susceptible to recolonization, unless new resources happen to be assigned to the site again, turning it into a new ephemeral patch. In a MELP system, the persistence of an individual strongly depends on its interpatch dispersal. This type of metapopulation system has important consequences for ecological and evolutionary aspects of the species that directly or indirectly depend on it (Harrison 1991, Hanski 1999, Altermatt and Ebert 2010).

For communities structured in a MELP, the ephemeral subpopulations established in patches are responsible for promoting emigration and, consequently, the colonization of other patches (Altermatt and Ebert 2010). Both the amount of resources per patch (ephemeral habitat size) and the frequency with which resources are allocated to the corresponding site (allocation probability) affect the dispersal of the species. Thus, the abundance and spatial positioning of the local populations are important characteristics of the system, determining the number of migrants while also being responsible for the dynamics (Altermatt and Ebert 2010), persistence and spatial distribution of the species involved. 
In the MELP context, the optimal choice of a feeding or oviposition patch is crucial for species persistence; once a patch is chosen, the exploitation of the resources should be maximized to improve the species' reproductive fitness. Parasitoid insects have been pinpointed by many empirical and theoretical studies as excellent biological models for studying foraging strategies (Godfray 1994, Outreman et al. 2005, Amat et al. 2009). The effectiveness of parasitoids is influenced by their capacity to find and attack hosts, and their reproductive success is directly related to their foraging strategies (Hassell 1978, Godfray 1994, Outreman et al. 2005). The decision of a female parasitoid to explore new patches and how to use its hosts for reproduction is related to its capacity to adopt an 'optimal' foraging strategy (Fretwell and Lucas 1970, Charnov 1976, Outreman et al. 2005). The consequences of parasitoid reproductive success are reflected directly in the demographic dynamics within a local patch, since the parasitoid's abundance affects host density and, consequently, its own persistence.

During patch exploitation, parasitoids can adopt different strategies depending on the density of conspecific competitors and hosts in the patch (Beddington et al. 1975, Godfray 1994, Hassell 2000, Grillenberger et al. 2009a). These conditions determine the 'quality' of the exploited patch (Fauvergue et al. 2006). Therefore, female parasitoids exhibit different behaviors according to their capacity to attack hosts and according to the level of patch quality.

In some parasitoid species that exhibit haplodiploid sex determination (arrhenotokous parasitoids), females can control the sex ratio of their offspring and can maximize their fitness by reducing the competition for mates between their sons in response to the foundress number (females that lay eggs) and the number of already parasitized hosts in a patch (Hamilton 1967, Shuker et al. 2006, Grillenberger et al. 2009a). The progeny sex ratio adjustment can be considered a strategy that female parasitoids use to maximize their fitness on exploited patches of hosts. This behavior can be an adaptive strategy for parasitoids that participate in a MELP, in which interactions occur over a short time and dispersion to other patches has risks of predation or even difficulties finding another profitable patch to explore.

In this study, we develop a mathematical model to understand how host-parasitoid populations are distributed over an environment consisting of sites where resources for the hosts are ephemeral and are randomly distributed among the sites at each generation (MELPs). Our main purpose is to evaluate how the coexistence of hosts and parasitoids in a MELP is influenced by 1) resource allocation probability (probability of resource appearance in a site), 2) variation in resource size (ephemeral patch size) and 3) number of sites available to receive resources in the system. To estimate the demographic and dynamic parameters of our model, we performed a series of laboratory experiments in which hostparasitoid interactions were investigated. For this, we studied a long-term interaction between a blowfly species, Chrysomya megacephala (Diptera: Calliphoridae), which uses ephemeral resources to aid juveniles' development, and Nasonia vitripennis (Hymenoptera: Pteromalidae), an arrhenotokous parasitoid of pupal-stage dipterans, in a laboratory microcosm. In the experimental section, we measured the persistence and local demographic processes of host-parasitoid interactions in two scenarios. In scenario 1, basal resources were constantly present at a single site, so species did not disperse to colonize new patches. In scenario 2, two sites were available, but resources were placed in only one site. At the end of each generation, the resources in the present patch expired (i.e. they were ephemeral) and new resources were placed in the other site, forcing the species to disperse and colonize the other site in order to survive.

\section{Material and methods}

\section{The study organisms}

Nasonia vitripennis (Hymenoptera: Pteromalidae) is a small gregarious parasitoid wasp that attacks the pupal stage of up to 68 dipteran host species (Whiting 1967). This parasitoid species is found in several habitats where flies are present; they parasitize a broad range of hosts, from ornithoparasitic bird blowflies in bird nests (Grillenberger et al. 2009a) to necrophagous dipterans carcasses (Grassberger and Frank 2004). Females lay clutches of eggs on host pupae. After emergence, female parasitoids become sexually mature after $48 \mathrm{~h}$ and begin laying eggs within $96 \mathrm{~h}$. Mating occurs at the locale of emergence because males are flightless and do not disperse to other patches. After mating, females must disperse to find new hosts because hosts are frequently not constant at a local site.

Laboratory experiments and field observations have shown that $N$. vitripennis modulates its progeny sex ratio according to environmental conditions, exhibiting female-biased sex ratios in favorable patches of exploitation (Hamilton 1967, Molbo and Parker 1996, Burton-Chellew et al. 2008, Grillenberg et al. 2009a). The adjustment of sex ratio can be considered a strategy adopted by female parasitoids to maximize their fitness on exploited patches of hosts, which has consequences for the host-parasitoid dynamics.

Chrysomya megacephala (Diptera: Calliphoridae) is a necrophagous blowfly species. Its reproduction is conditioned to the presence of carrion, where their larvae can feed until they become pupae around it. This food resource is ephemeral: newly emerged adult flies must look for other food resources to continue reproduction. In this way, the interaction between $N$. vitripennis and C. megacephala pupae occurs within a period of one generation in an ephemeral patchy resource.

\section{Laboratory microcosm}

The species used in this study were collected in the vicinity of the Campus of Universidade Estadual Paulista, Botucatu, São Paulo, Brazil and were maintained in laboratorycontrolled conditions $\left(25^{\circ} \mathrm{C}, 70 \% \mathrm{RH}, 12: 12\right.$ light:dark cycle).

The laboratory microcosm was used to experimentally investigate the effects of parasitism, changes in the parasitoid progeny sex ratio and host-parasitoid dispersal rates on the colonization of ephemeral patches and the time of host-parasitoid coexistence. Experimental arenas were designed to simulate the natural scenario: $C$. megacephala adult blowflies lay eggs on given resources, and after hatching, larvae feed 
and reach pupal stage, thus becoming susceptible to parasitism by $N$. vitripennis.

The observed life cycle of $C$. megacephala consists of approximately three days as eggs, 1 st and 2 nd instar larvae, and two days as 3rd instar larvae and four days as pupae. After adult emergence and mating, it takes seven days for the flies to start laying eggs. The life cycle observed for $N$. vitripennis is as follows: larvae hatch approximately $36 \mathrm{~h}$ after egg deposition and exhibit three developmental life stages, becoming pupae after approximately nine days. The pupal stage lasts for three days, with adult emergence following the chewing through the puparium, which occurs 14 days after hatching. The reproductive periods of parasitoids are always synchronized with the susceptible period of the blowfly pupae.

We used two different types of experimental arenas to analyze the roles of colonization of ephemeral resources by interacting species. In scenario 1 , a single site is considered and the resources are replenished at the end of each generation, so that the individuals do not disperse to reproduce. In scenario 2, the resources are removed from the site after one life cycle, so that the individuals must disperse to the nearby patch, where fresh resources have been added, in order to reproduce. Glass boxes $(60 \times 50 \times 40 \mathrm{~cm})$ represented sites. Glass boxes with $200 \mathrm{~g}$ of ephemeral resources (ground beef) represented a susceptible patch to be colonized by the host and, consequently, by parasitoids. This fixed amount of resource allowed limitation of the host population increase in a colonized patch. The definition of the box carrying capacity is shown in Appendix 1 (Fig. A1.1). For all sites, a honeywater solution (1:3) was available to feed adult species, and wood shavings were available for blowfly pupation. Seven replicates were performed for each experimental arena.
The numbers of individuals emerging from each site box were recorded to obtain the host and parasitoid population sizes per generation until one of species became extinct. Microcosms were maintained under the controlled environmental conditions previously mentioned.

\section{Scenario 1: colonization without species dispersal}

Initially, 300 blowfly larvae and four mated $N$. vitripennis females were introduced into the patch boxes (sites with ephemeral resources). Four days later, the majority of blowfly larvae had pupated; all blowfly pupae were removed and placed individually into transparent gelatin capsules. The remaining parasitoids and resources were also removed.

The blowflies were censused as they emerged from the capsules. Male and female flies were counted and subsequently released into the birth box to feed and mate. After seven days, new resources were introduced into the box to allow the blowflies to lay eggs. We allowed the blowflies to lay eggs on the available resources for one day, after which the adult blowflies were removed and only juveniles (progeny) were left feeding on the resources. This period frequently coincided with the emergence of parasitoids from the parasitized blowfly pupae that remained in capsules. We censused male and female parasitoids from each capsule (to quantify sex ratio) and then released the parasitoids into the same box as the blowflies (the birth box). This protocol allowed the numbers of individuals in each generation to be recorded. The experimental setup simulated a system in which ephemeral resources are constantly present, so that the individuals do not need to disperse to look for new patches. This procedure was repeated until one of the two species became extinct (Fig. 1a-e).
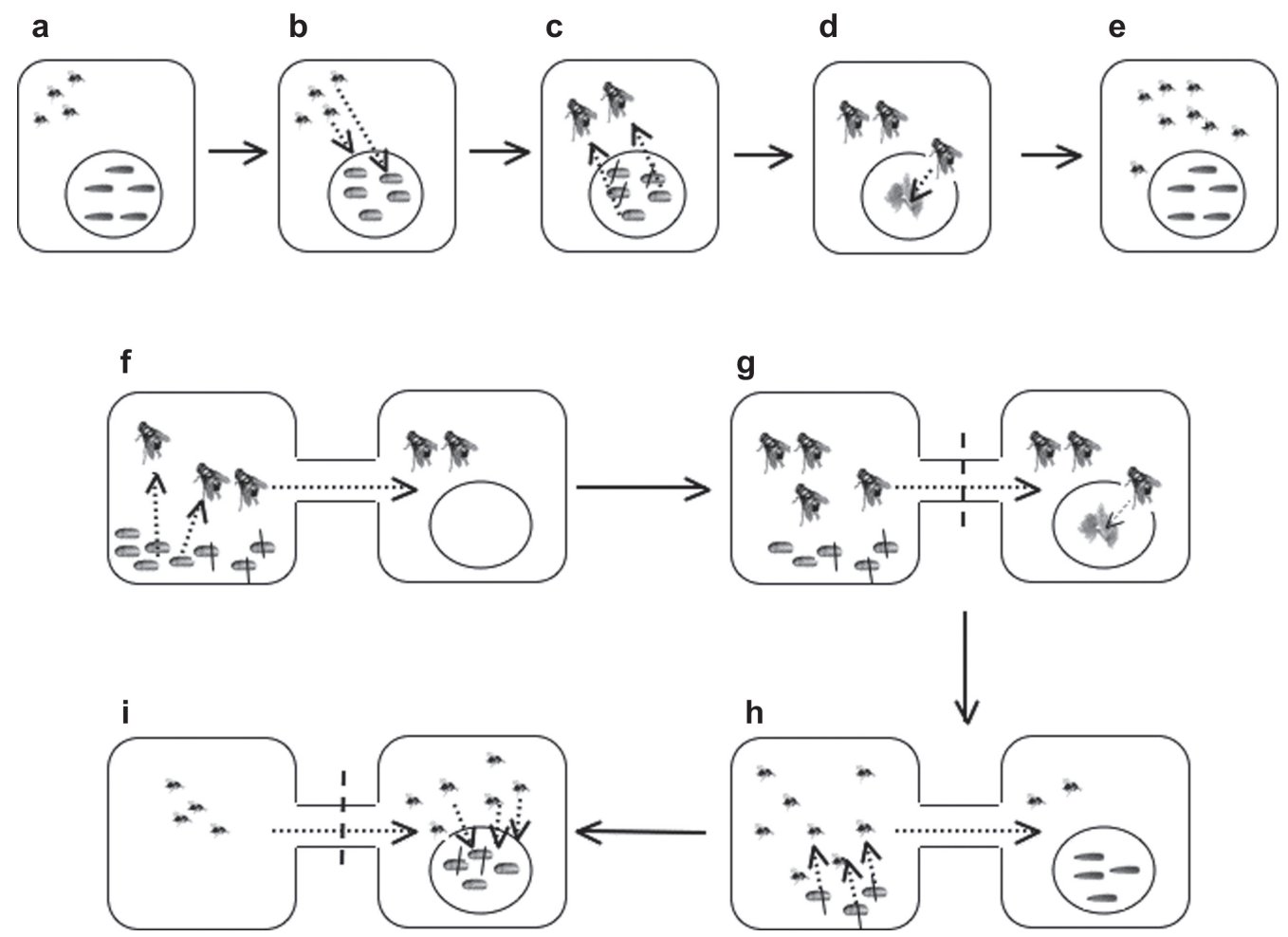

Figure 1. Schematic diagram of the experimental laboratory microcosm protocol. Scenario 1: constant presence of ephemeral resource $(\mathrm{a}-\mathrm{e})$; scenario 2: variation of resource presence (colonization requires dispersion of species) $(\mathrm{f}-\mathrm{i})$. 


\section{Scenario 2: colonization by species dispersion}

In this experiment, we used two glass boxes connected by a corridor (a glass tube, $5 \times 15 \mathrm{~mm}$ ) that was placed in the center of one wall of the boxes to permit the species to disperse between the two boxes. One of the boxes was initialized as described in scenario 1 above, whereas the second box was kept empty (without resources) until the emergence of adult insects. The procedures for removal, census and release of populations were the same as described above; however, the new batch of ephemeral resources was always introduced into the previously empty box (thus creating a new patch). For 24 $\mathrm{h}$, the corridor (tube) was opened to allow the blowflies to disperse. After $24 \mathrm{~h}$, the dispersed blowflies were removed, and the populations in the two boxes (with and without resources) were censused to obtain the host dispersal rate. Only juveniles that had hatched from the dispersed blowfly eggs were left to feed on the resources. The 24-h period was chosen to facilitate the synchronization of the populations, because the blowfly larvae hatched approximately $24 \mathrm{~h}$ after the eggs were laid. This procedure ensured that the blowfly juveniles all had similar ages, such that they reached the pupal stage and became susceptible to parasitoids at approximately the same time.

Parasitoid emergence occurred frequently in the final blowfly larval instar (three days after hatching). We allowed the parasitoids to be in contact with their hosts from their emergence until the end of the susceptible period of the hosts. To estimate parasitoid dispersal rates, the corridor between patches was opened for parasitoid dispersion for two hours per day over the course of six days (covering the last larval stage and the pupal period of the blowflies). During this period, the parasitoids that dispersed to the new patch were observed and recorded. After parasitoid dispersal, the blowfly pupae were removed from the new patch, placed into transparent gelatin capsules and later released back into the same box as adult (host or parasitoid) insects. The entire procedure was repeated until one of the two species became extinct (Fig. 1f-i).

Limitation of the time that the corridor was opened for species dispersal was necessary for several reasons: 1 ) to ensure that dispersal was indeed driven by ephemeral resources; 2) to ensure that the populations of the two boxes did not mix into a single population (French and Travis 2001, Bonsall et al. 2002); and 3) to facilitate the population censuses (by dealing with insects in the same life stage) and thereby enable the analyses of demographic processes proposed in this study.

\section{Statistical analyses}

We calculated the averages, medians and variances of coexistence times in the two scenarios as described below; we used the population census of each box as replicates.

\section{Variation in the number of parasitized hosts in a patch}

We tested if the number of parasitized host pupae $\left(H_{p}\right)$ in a patch (at the end of each generation that we observed coexistence) varied with changes in host and female parasitoid densities. We used two-way ANOVAs to compare the $\ln$ (mean of parasitized hosts +1 ) variations among patches.
Variation in progeny sex ratio of parasitoids in a patch

At the end of each generation in which we observed coexistence, we tested if the sex ratio of parasitoid offspring (proportion of males) in a patch changed with parasitized host density $\left(H_{p}\right)$ and with the variations in the proportion of parasitoid foundresses per host $(F / H)$. We used two-way ANOVAs to compare the proportion of male variation among patches after arcsin square-root transformation.

\section{Host and parasitoid dispersal rates}

We calculated the numbers of dispersed individuals in scenario 2. We tested whether host and parasitoid dispersals were density-dependent. We used generalized linear models with negative binomial regression errors and log-link function. We regressed the number of hosts and/or parasitoids that dispersed against the density in the native site (from which dispersers originated).

For all analyses, we first constructed full models, including all significant terms, to generate minimal adequate models following backward stepwise deletion (Crawley 2007). Significances were tested using likelihood ratio tests to compare the changes in model deviance after deleting each term. All analyses were performed using R ver. 2.12 (The R Foundation for Statistical Computing, < www.R-project.org >).

\section{Role of demographic processes in empirical host-parasitoid interaction}

The most striking difference between the results of our controlled experiments and the dynamics of hosts and parasitoids in natural environments is that one of the species always went extinct in the former, whereas coexistence is often found in the latter. Nonetheless, the experiments can help us understand the role of demographic factors in the persistence of these interacting species in nature. Later in this paper we develop a mathematical model based on these results. The model shows how these demographic factors, when embedded in a large spatial structure, can influence species dynamics and lead to coexistence. The model was not designed to reproduce the experimental data in detail. Rather, this model was meant to incorporate the demographic effects observed in the experiments and to explore our hypothesis that species persistence can only occur in a spatially explicit environment.

In constructing the model, we had to choose mathematical functions describing functional response, changes in the parasitoid sex ratio and dispersal rates of hosts and parasitoids. The demographic effects observed in the laboratory microcosm were used to define these functions, which were chosen to be as simple as possible. For fitting the functions to the experimental data, we used the 'nls' package of $\mathrm{R}$ statistical software (ver. 2.12). Because we were quantifying the behavior of many individuals, we expected high variability in the experimental data. Thus, to ensure a good representation of the data, we compared the averages of the experimental data with the function adjustment as given by the statistical software (Appendix 2 Fig. A2.1-3). The experimental procedures and the details of the fits to the empirical data are shown in Appendix 2. 
Table 1. Estimated parameters for the effect of parasitoid foundress $\left(F_{o}\right)$ and available host $\left(H_{o}\right)$ densities on parasitism (in terms of parasitized hosts) by Nasonia vitripennis in a patch.

\begin{tabular}{lccc}
\hline & Estimate $( \pm \mathrm{SE})$ & t-value & $\mathrm{p}$ \\
\hline Intercept & $1.92( \pm 0.022)$ & 8.57 & $<0.001^{*}$ \\
$F_{o}$ & $3.9 \times 10^{-4}\left( \pm 8.5 \times 10^{-5}\right)$ & 4.57 & $<0.001^{*}$ \\
$H_{o}$ & $3.34 \times 10^{-3}\left( \pm 3.92 \times 10^{-4}\right)$ & 8.52 & $<0.001^{*}$ \\
\hline
\end{tabular}

\section{Results}

The averages $( \pm \mathrm{SE})$, medians and variances of coexistence times were 3.28 ( \pm 2.05$), 2.0$ and 3.63 generations, respectively, for scenario 1 ; they were 2.86 ( \pm 1.77 ), 2.0 and 2.69 generations, respectively, for scenario 2 . As expected, the data displayed high variability in both scenarios because we did not control the variation in host and parasitoid densities in the boxes. Hosts went extinct before parasitoids in all replicates in scenario 1 . In scenario 2, hosts went extinct before parasitoids in four of seven replicates $(57.1 \%)$; in the remainder of the replicates ( $42.9 \%$ ), parasitoids went extinct and hosts persisted. Long-term coexistence of hosts and parasitoids was never observed, indicating that the C. megacephala $-N$. vitripennis interaction is prone to extinction. More importantly, the results of scenario 2 suggest that coexistence can be attained in a larger spatial structure because host populations can escape parasitism by colonizing patches not yet reached by parasitoids.

\section{Variation in numbers of parasitized hosts in a patch}

The analyses of the results indicated that host $\left(H_{o}\right)$ and female parasitoid $\left(F_{o}\right)$ density effects were positively correlated with changes in the numbers of parasitized hosts $\left(H_{p}\right)$ $\left(\mathrm{F}_{2,41}=39.1, \mathrm{p}<0.001 ; \mathrm{F}_{2,41}=72.6, \mathrm{p}<0.01\right.$, respectively; Table 1).

\section{Variation in progeny sex ratio of parasitoids in a patch}

Our results indicated significant interaction effects from the proportions of conspecific competitors per host $\left(F_{o} / H_{o}\right)$ and number of parasitized hosts $\left(H_{p}\right)$ on the progeny sex ratio adjustment of $N$. vitripennis foundresses $\left(\mathrm{F}_{3,38}=5.35\right.$, $p=0.003$ ). This interaction effect was positively correlated with an increase in male progeny, meaning that the increase of competitors per host density and the decrease of hosts available increased the sex ratio in the parasitoid progeny (Table 2).

Table 2. Estimated parameters for the effects of the proportion of parasitoid foundresses per available host $\left(F_{o} / H_{o}\right)$ and the density of parasitized hosts $\left(H_{p}\right)$ on progeny sex ratio adjustment of Nasonia vitripennis in a patch.

\begin{tabular}{lccc}
\hline & Estimate $( \pm \mathrm{SE})$ & t-value & $\mathrm{p}$ \\
\hline Intercept & $4.6 \times 10^{-1}( \pm 0.034)$ & 13.6 & $<0.001$ \\
$F_{o} / H_{o}$ & $-1.35 \times 10^{-2}( \pm 0.008)$ & -1.6 & 0.12 \\
$H_{p}$ & $2.3 \times 10^{-4}\left( \pm 0.5 \times 10^{-4}\right)$ & 2.7 & $0.009^{*}$ \\
$F_{o} / H_{o} \times H_{p}$ & $1.14 \times 10^{-4}\left( \pm 0.5 \times 10^{-4}\right)$ & 2.143 & $0.038^{*}$ \\
\hline
\end{tabular}

Table 3. Estimated parameters for the effect of the density of individuals at the origin site on host (a) and parasitoid (b) dispersal rates ( $p$ values are given using the Wald-test). $H_{o}=$ host density and $F_{o}=$ foundress density at the native site.

\begin{tabular}{lcrc}
\hline & Estimate $( \pm \mathrm{SE})$ & z-value & $\mathrm{p}$ \\
\hline (a) Intercept & $2.03( \pm 0.28)$ & 7.09 & $<0.001^{*}$ \\
$H_{o}$ & $0.008( \pm 0.001)$ & 6.03 & $<0.001^{*}$ \\
(b) Intercept & $4.10( \pm 0.31)$ & 13.19 & $<0.001^{*}$ \\
$F_{o}$ & $0.6 \times 10^{-4}\left( \pm 0.12 \times 10^{-3}\right)$ & 5.32 & $<0.001^{*}$ \\
\hline
\end{tabular}

\section{Host and parasitoid dispersal rates}

The host density at the native site $\left(H_{0}\right)$ showed significant effects on host dispersal rates $\left(\chi^{2}=14.521, \mathrm{DF}=17\right.$, $\mathrm{p}<0.001$, Table $3 \mathrm{a}$ ). The dispersion of hosts was positively correlated with the host density at the native site.

For analyses of parasitoid dispersal rates, we used data from five replicates because in two experimental arenas, the migration of parasitoids did not occur. The female parasitoid density at the native site $\left(F_{o}\right)$ showed significant positive effects on foundress dispersal rates $\left(\chi^{2}=16.97, \mathrm{DF}=13\right.$, $\mathrm{p}<0.001$, Table $3 \mathrm{~b}$ ).

\section{Modeling host-parasitoid dynamics in MELP}

In this section, we describe the development of a mathematical model for the dynamics of $C$. megacephala and $N$. vitripennis interactions. The modeling of the withinpatch dynamics considered the effects of demographic factors found in our empirical results, and the functions describing these effects were adjusted to the experimental data described in the previous sections. The mathematical model can extrapolate the system from one or two sites to a large number of connected sites, mimicking natural environments and allowing the investigation of coexistence. The motivation for the model comes both from the literature (Baumgartner and Greenberg 1984, Grillenberger et al. 2009b) and from the results obtained in the second experimental scenario, which showed that the dispersal ability of the parasitoids was lower than that of the hosts. Parasitoids were weaker dispersers both in terms of the fraction of individuals that dispersed and in terms of their average range of dispersal. In our experiments, this led to situations where the parasitoids did not reach the newly available patch, leaving unparasitized hosts free to persist there. This suggested that, in a multi-connected patch system, hosts and parasitoids can reach a state of coexistence through the occurrence of patches with low densities of parasitoids. The model was first tested to corroborate our empirical observations that species coexistence is not possible in the experimental setup described in the previous sections (scenarios 1 and 2). We then confirmed that the model can simulate coexistence in a sufficiently large spatially structured landscape.

\section{Variable basal resources in MELP}

We considered a metapopulation of hosts and parasitoids distributed in a landscape with a large number of cells connected by migration. We considered arenas of several sizes 
and found that the qualitative results for coexistence and spatial patterns were not sensitive to metapopulation sizes and/or boundary effects for arenas larger than $20 \times 20$ sites. The majority of our simulations were conducted in a $50 \times 50$ arena. Each cell represented a discrete spatial unit, also termed a 'site' (a place available to receive ephemeral resources in MELP). Resources were randomly distributed to the sites at the beginning of each generation, thus transforming sites into patches with a probability, $p r$ (termed allocation probability in this study). Resources were removed at the end of each generation, after which a new random distribution of resources took place. The presence of resources at a site did not imply that the site was automatically occupied by hosts; occupation depended upon colonization by individuals from other sites. The presence of resources at a site also did not affect the site's probability of receiving resources at a later time. Our simulations analyzed the following two situations: 1) fixed size of ephemeral basal resources (carrying capacity $k=1600$ host individuals per patch) and 2) variable sizes of ephemeral basal resources (the carrying capacity assigned to a patch was randomly selected in the interval $1600 \leq k_{\text {rand }} \leq 8000$ host individuals).

In the simulations, we first considered a homogeneous situation in which resources were allocated to all sites $(p r=1)$. We compared these results with simulations in which $p r<1$, progressively decreasing $p r$ until no resources were available in the landscape. At each time step, we calculated the average number of hosts, parasitoids and ephemeral resources. The dynamics of host-parasitoid interactions are described in the next section.

\section{Host-parasitoid patchy population dynamics}

Our aim is to understand how variation in size of ephemeral basal resources $\left(R_{t}\right)$ and its frequency of distribution per site $(p r)$ influence the host-parasitoid dynamics in a spatially explicit landscape.

\section{Patch colonization}

Only hosts $(H)$ and female parasitoids $(F)$ are allowed to disperse to new patches. Because male parasitoids $(M)$ are not capable of dispersing, mating occurs only at the native site. After the emergence of adults, blowflies and female parasitoids look for new substrate on which to reproduce. If new resources appear at the site of birth, the decision to disperse is based on the density of conspecifics (in the case of blowflies) and on local host abundance (in case of parasitoids). Female parasitoids adjust their progeny sex ratio based on patch quality, quantified by the ratio $F / H$. Part of the new host generation, which originates from non-parasitized hosts, and newly born female parasitoids disperse and colonize the patches around their home site. However, blowflies and parasitoids are allowed to visit only one patch in their life cycle. This allows us treat the species abundance in each patch as a real local population, defined as discrete groups in which mating, reproduction and interactions take place (Harrison 1991).

\section{Host-parasitoid interaction in an ephemeral patch}

Hosts and parasitoids interact according to Eq. 1 below. $H_{i, t}$ $F_{i, t}$ and $M_{i, t}$ represent the populations of blowflies, female and male $N$. vitripennis on patch $i$ and time $t$, respectively. Time is discrete and measured in numbers of generations. Host and parasitoid populations are considered extinct for population sizes lower than 0.1 individuals per patch. The local dynamics in each patch consist of blowflies laying eggs, their larvae feeding and becoming pupae and adult parasitoids searching for blowfly pupae to parasitize. The parasitoid life cycle is assumed to be synchronous with that of the host, as was observed in the empirical section.

The equations describing the within-patch interactions are given below. We use small letters for the populations at generation $t+1$ because these are not yet the final populations. We switch back to capital letters after dispersal is taken into account:

$$
\begin{aligned}
& h_{i, t+1}=H_{i, t}\left(\frac{\lambda k}{H_{i, t}(\lambda-1)+k}\right)\left[1-p\left(H_{i, t}, F_{i, t}\right]\right. \\
& f_{i, t+1}=c H_{i, t} p\left(H_{i, t}, F_{i, t}\right) s\left(H_{i, t}, F_{i, t}\right) \\
& m_{i, t+1}=c H_{i, t} p\left(H_{i, t}, F_{i, t}\right)\left[1-s\left(H_{i, t}, F_{i, t}\right)\right]
\end{aligned}
$$

The population of blowflies increases with intrinsic growth rate $\lambda$ (assumed to be 1.5) and reaches the carrying capacity $k$ (assumed to be fixed at $k=1600$ or variable between $1600 \leq k_{\text {rand }} \leq 8000$ host individuals) when parasitoids are absent.

The effect of parasitism is included in the function 1 $p(H, F)$, which gives the proportion of blowflies surviving parasitism (defined in Appendix 2.1). The population of female $N$. vitripennis in the next generation is given by the number of parasitized blowflies, $H_{i, t} p(H, F)$, multiplied by $c$, the average number of adult parasitoids emerging from each host (assumed to be 20). The model does not consider superparasitism (one host parasitized by more than one parasitoid); however, the value adopted for the numerical response of $N$. vitripennis can represent the number of parasitoids laid by more than one female in a one-host puparia.

The functional response $p(H, F)$, representing the fraction of parasitized blowfly pupae, is defined as:

$$
p(H, F)=\left\{\begin{array}{lll}
1 & \text { if } & v(H, F)>1 \\
v(H, F) & \text { if } & v(H, F) \leq 1
\end{array}\right.
$$

where

$v(H, F)=\frac{32 \alpha F}{H+\beta F^{2}}$

and $\alpha$ is the parasitoid attack rate. The term $32 \alpha$ represents the maximum number of pupal hosts that one parasitoid can parasitize if there was no competition for hosts among conspecifics (Fig. A2.1a). The parameter $\beta$ represents the level of competition interference and reduces the female's potential to attack hosts in depleted patches. This is caused mainly by the delay between successive female ovipositions, due to the high probability of encountering hosts that have already been parasitized. The effects of this competition interference increase with the density of females (foundresses) in the patch (Fig. A2.1b). From our empirical data, we estimated the values of $\alpha=0.34$ and $\beta=0.06$. 
The function $s(H, F)$ defines the proportion of female $N$. vitripennis that emerged and was controlled by the progeny sex ratio adjustment (defined in Appendix 2.2). The population of male parasitoids is similar, but multiplies $1-s(H, F)$. We use

$$
s\left(H_{i, t}, F_{i, t}\right)=\exp \left(\frac{-\gamma F_{i, t}}{H_{i, t}}\right)
$$

where $\gamma$ represents the degree of sex ratio adjustment. This functional form reflects the proportion $F / H$ (quality of patch) necessary to provoke changes in the progeny sex ratio by a female parasitoid. Indeed, the larger the proportion $F / H$, the better it is for a female to increase the number of male offspring, as this increases the chances for its sons to mate with daughters of other females. By adjusting this function to our empirical data, we obtained $\gamma=0.3$ (Fig. A2.2).

\section{Dispersal}

The landscape is represented by a square grid with reflective boundaries, and the ephemeral subpopulations occupying individual patches are coupled by distance-dependent dispersal. After the new generations of blowflies and parasitoids have emerged, the original resources available to hosts disappear, and the populations have to disperse to find fresh ephemeral resources to reproduce. Fresh ephemeral resources are allocated randomly, as explained earlier. Dispersal occurs within a distance $R_{\text {dist }}$ of the original patch, so that the dispersal area is approximately $\pi R_{\text {dist }}^{2}\left(R_{\text {dist }}=6\right.$ in our simulations, for both blowflies and parasitoids). However, not every site in the dispersal area is a patch (i.e. a site with resources). We model the fraction of blowflies that leaves the current patch $\left(h_{i, t+1}^{\text {out }}\right)$ by

$$
h_{i, t+1}^{\text {out }}= \begin{cases}\mu_{H} \frac{h_{i, t+1}^{2}}{h_{i, t+1}+100} & \text { if } \quad R_{i, t+1}>0 \\ h_{i, t+1} & \text { if } \quad R_{i, t+1}=0\end{cases}
$$

where $\mu_{H}$ is the maximum dispersal rate of hosts in a patch with high density of blowflies (density-dependent dispersion) and $R_{i, t+1}$ is the amount of resources in the originating site $i$ at time $t+1$. The value of $\mu_{H}$ was fixed at 0.85 , given by adjustment of the dispersal function to the empirical data (Appendix 2.3, Fig. A2.3a). The $h_{i, t+1}^{\text {out }}$ host population disperses among neighboring patches inside the dispersal area of radius $R_{\text {dist }}$. Each patch $j \neq i$ receives a fraction of these host individuals, depending on their distance $r_{i j}$ to the patch of origin:

$$
h_{j, t+1}^{i n}=\left\{\begin{array}{lll}
\frac{0.05}{r_{i j}} h_{i, t+1}^{\text {out }} & \text { if } & R_{j, t+1}>0 \\
0 & \text { if } & R_{j, t+1}=0
\end{array}\right.
$$

If the number of patches in the dispersal area is not large enough to absorb all the dispersers, the remaining $h_{i, t+1}^{\text {out }}$ die. After the dispersal phase, the population of blowflies in each patch is given by:

$$
H_{i, t+1}=h_{i, t+1}-h_{i, t+1}^{\text {out }}+\sum_{r_{i, j \leq R_{H}}} h_{i, t+1}^{\text {in }}
$$

The dispersal of the parasitoids occurs after that of the hosts. This is because it takes longer for $N$. vitripennis to reach the adult stage, as observed in the experiments. We modeled the number of dispersing female parasitoids as depending on the density of hosts in the originating patch:

$$
f_{i, t+1}^{\text {out }}= \begin{cases}\mu_{F}\left(\frac{300}{300+H_{i, t+1}}\right)\left(\frac{f_{i, t+1}^{2}}{f_{i, t+1}+100}\right) & \text { if } H_{i, t+1}>0 \\ f_{i, t+1} & \text { if } H_{i, t+1}=0\end{cases}
$$

Here, $f_{i, t+1}^{\text {out }}$ is the female parasitoid population that disperses to the neighboring patches in the dispersal area $R_{\text {dist }}$, and $\mu_{F}$ is the maximum parasitoid dispersal rate when the quality of the patch is bad (high-proportion $F / H$ ). The value of $\mu_{F}$ used in our simulations and obtained from empirical data is 0.4 (Appendix 2.3, Fig. A2.3b).

The female parasitoids $f_{i, t+1}^{\text {out }}$ disperse from patch $i$ to neighboring patches $j$ within a distance $r_{i, j} \leq R_{\text {dist }}$ only if there are hosts in patch $j$. In this case

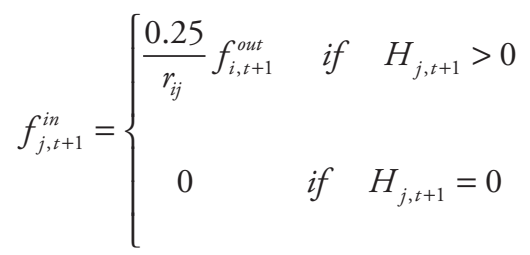

If the number of patches occupied by hosts is not large enough to absorb all dispersing parasitoids, the remaining $f_{i, t+1}^{\text {out }}$ die. After dispersal, the parasitoid population in each patch is given by

$$
F_{i, t+1}=f_{i, t+1}-f_{i, t+1}^{o u t}+\sum_{r_{i, j} \leq R_{F}} f_{i, t+1}^{i n}
$$

Comparing Eq. 5 and 8 we note that the fraction of dispersing hosts that stay on a nearby patch is 0.05 , whereas this fraction is 0.25 for the parasitoids. This accounts for the lower dispersal range of the parasitoids (Grillenberger et al. 2009 b), which will colonize only four new patches on average, whereas hosts may colonize up to 20 patches.

\section{Results: host-parasitoid MELP dynamics}

To determine whether the within-patch dynamics of the model represented the empirical findings, we first investigated the possibility of host-parasitoid coexistence in a non-spatial structure, as in experimental scenario 1, and for 

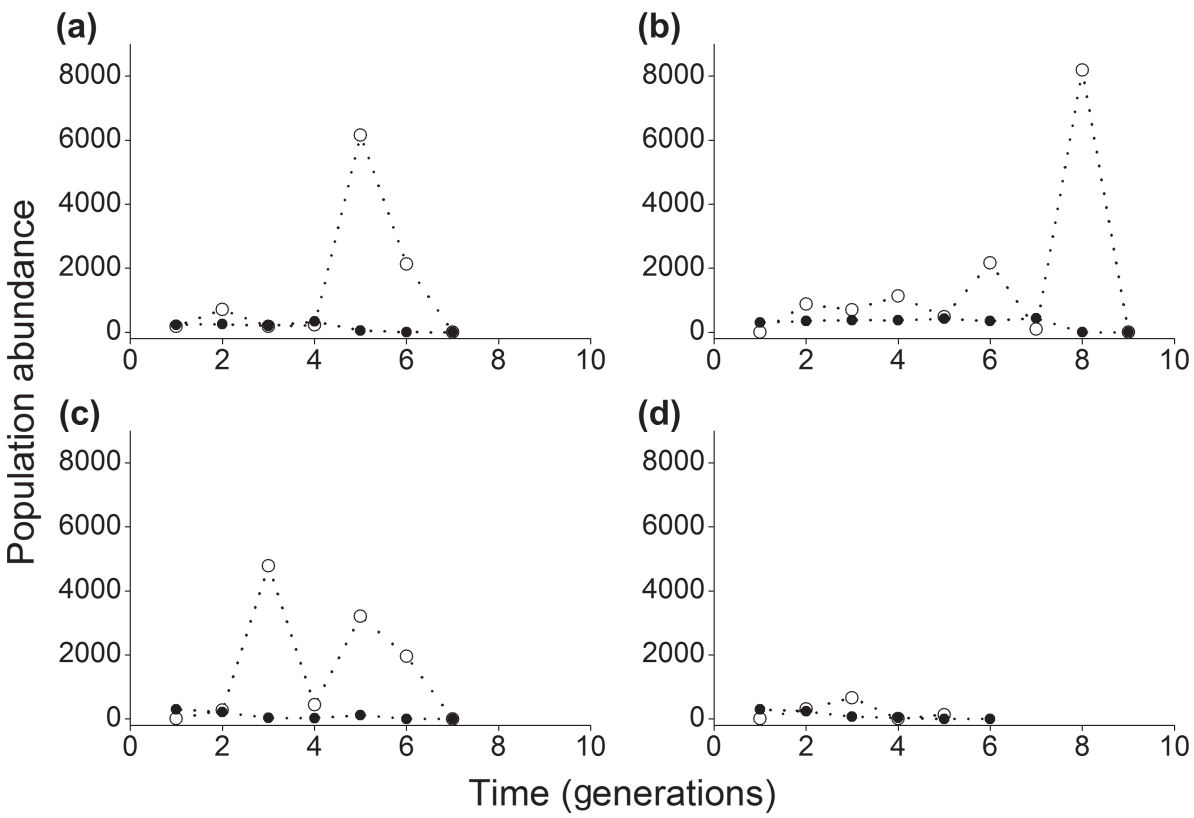

Figure 2. Host-parasitoid time series for: (a) representative experimental population sizes for scenario 1; (b) theoretical dynamics for scenario 1; (c) representative experimental population sizes for scenario 2; and (d) theoretical dynamics for scenario 2. Black circles represent C. megacephala (host), and white cycles represent $N$. vitripennis (parasitoid) populations. Parameter values: $\lambda=1.5 ; k=1600 ; \alpha=0.34 ;$ $\beta=0.06 ; c=20 ; \gamma=0.3$.

a two-site system, as in scenario 2 . In both cases, we used equation 1 to describe the dynamics. In the first case, the subsequent generation of hosts and parasitoids $\left(H_{t+1}\right.$ and $\left.F_{t+1}\right)$ was obtained directly from the individuals surviving from the host-parasitoid interaction, whereas in the second case, the subsequent generation was defined by the number of dispersers arising from the host-parasitoid interaction (fixed $\mu_{H}=0.85, \mu_{F}=0.4$ ). In both cases, the simulations resulted in species extinction over a time frame comparable to that of the experiments (Fig. 2). For this set of parameters, however, we did not observe the persistence of hosts, as observed experimentally in scenario 2 .

To test the importance of the metapopulation structure for the persistence of the species, we ran the host-parasitoid MELP model for 10000 time-steps for several sizes of the spatial grid and different frequencies of resource distribution per site $(p r)$. We fixed the resource carrying capacity $(k)$ at 1600 host individuals, which was the value used in the experimental sets. We started from a small square lattice with $10 \times 10$ sites, such that the dispersal range included all sites of the landscape, up to a lattice with $100 \times 100$ sites, in which the dispersers could reach a maximum of 120 nearest sites $\left(R_{H}=R_{F}=6\right)$. For small lattices, only host persistence was observed, and only for large values of $p r$. Host-parasitoid coexistence was found only for lattices larger than $20 \times 20$ and large values of $\operatorname{pr}$ (Fig. 3).

To understand the influence of basal resource size on hostparasitoid MELP, we explored the effects of fixed and random carrying capacities $\left(k=1600\right.$ and $1600 \leq k_{\text {rand }} \leq 8000$ host individuals) in a $50 \times 50$ lattice. In these cases, the progressive increase of $p r$ had important implications for persistence and distribution of species in the landscape. For both cases, coexistence was found for $p r \geq 0.6$ (Fig. 4), with large values of $p r$ increasing the host-parasitoid abundances and changing the dynamics and the spatial pattern distribution.
For fixed resource sizes (Fig. 4a) and $p r<0.4$, both, host and parasitoid populations went extinct and only the resources are represented as dark points. For $p r=0.5$, the parasitoids went extinct but the hosts survived and attained relatively large populations, limited only by carrying capacity. For $p r>0.5$, hosts and parasitoids coexisted, and their mutual interference caused large fluctuations around low abundances. However, for variable resource sizes and $p r \geq 0.7$, both host and parasitoid populations became abundant, with the latter fluctuating above the resource population size (Fig. $4 \mathrm{~b}$ ). These variable dynamics have implications for species occupancy and abundance distributions in the landscape. Larger values of the resource

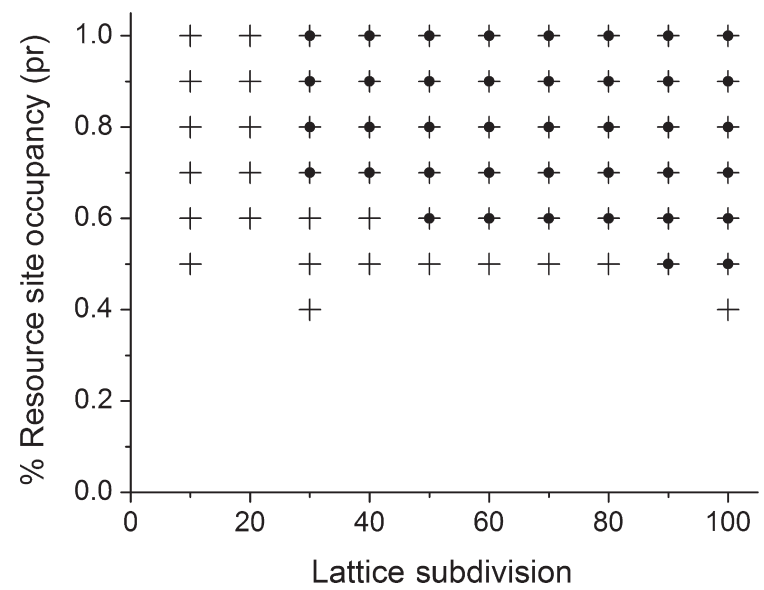

Figure 3. Theoretical host persistence $(+)$ and host-parasitoid coexistence $(\bullet)$ in a spatially structured environment for different lattice subdivisions and probabilities of resource allocation $(p r)$. Parameter values: $\lambda=1.5 ; k=1600 ; a=0.34 ; \beta=0.06 ; c=20$; $\gamma=0.3 ; \mu_{H}=0.85, \mu_{F}=0.4$. 

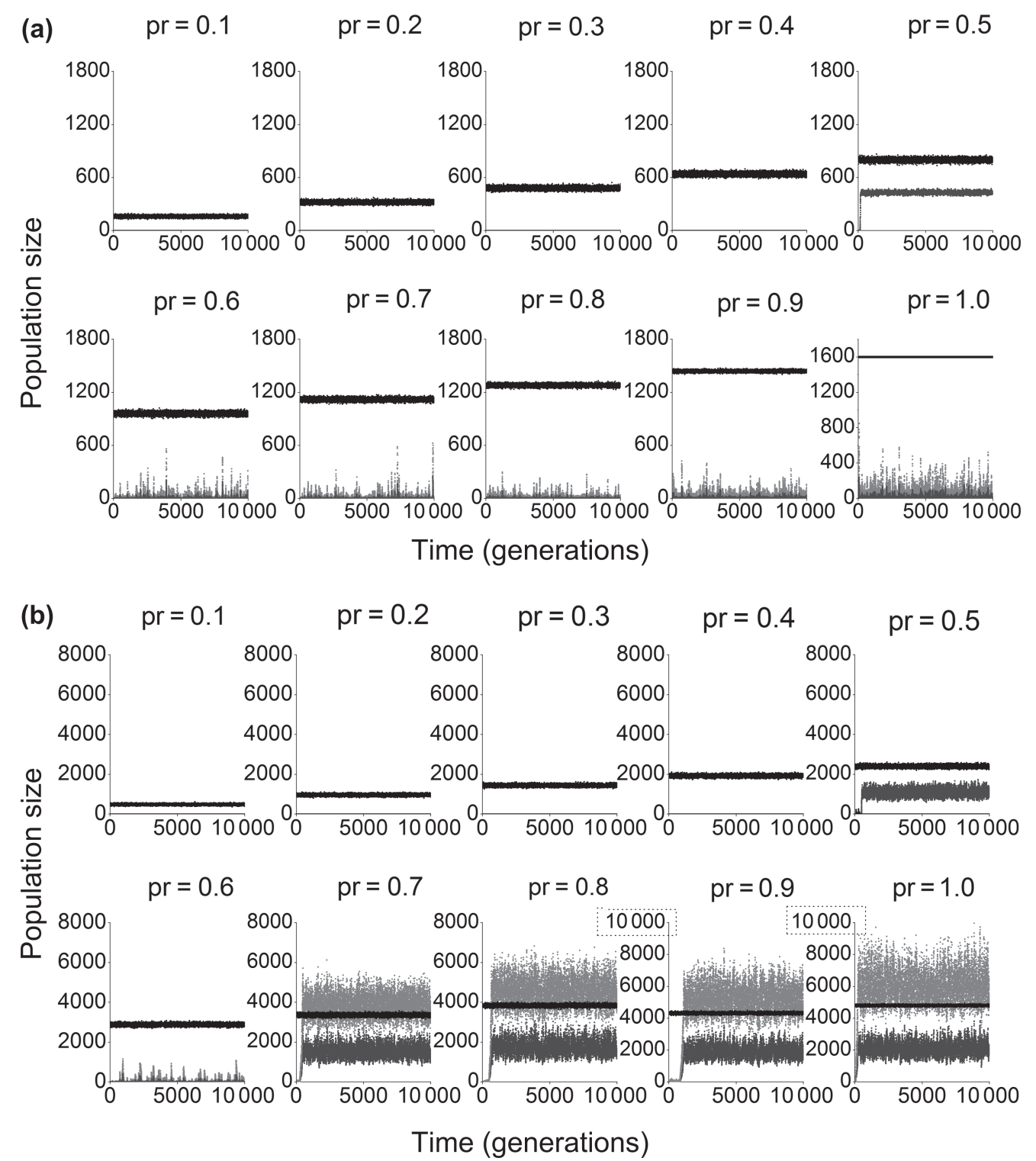

Figure 4. Persistence of $C$. megacephala and its parasitoid $N$. vitripennis over a grid of $50 \times 50$ sites as a function of time and reflective boundary conditions for different probabilities of ephemeral resource site occupancy $(p r)$ in a spatially structured landscape. (a) Fixed size of the ephemeral resource ( $k=1600$ host individuals) and (b) variable ephemeral resource size $\left(1600 \leq k_{\text {rand }} \leq 8000\right.$ host individuals). Black, dark gray and light gray symbols represent, respectively, the basal ephemeral resource, host and parasitoid abundances. Parameter values: $\lambda=1.5 ; \alpha=0.34 ; \beta=0.06 ; c=20 ; \gamma=0.3 ; \mu_{H}=0.85, \mu_{F}=0.4$.

size $(k>1600)$ were also analyzed, and the same qualitative patterns were obtained.

Figure $5 \mathrm{a}$ and $5 \mathrm{~b}$ show plots of the species landscape occupancy for different values of $p r$ for fixed and variable resource sizes, respectively. As previously mentioned, coexistence was found at $p r \geq 0.6$. Figure 5a shows that the number of patches occupied by both hosts and parasitoids increased with increasing $p r$. However, patches with hosts and no parasitoids were found at all values of $p r$. In these cases, we observed large population fluctuations leading to traveling waves of host and parasitoid abundances, as shown by the images in Fig. $6 \mathrm{a}$ for times 9990, 9995 and 10 000. In the first panel, patches with a high density of hosts are apparent, whereas in the second panel, the parasitoid population in those same patches is larger than the host population (a case of overexploitation). In the third panel, the parasitoid population decreases, due to intense parasitism at previous timepoints. For large values of the (fixed) carrying capacity $(k=8000)$, patches with hosts and no parasitoids did not occur.

The case of variable resource sizes $\left(1600 \leq k_{\text {rand }} \leq 8000\right)$ displayed a similar behavior, with the difference that patches with host and no parasitoids did not occur for large values of $p r$ (Fig. 5b). In these cases, the parasitoid metapopulation divided in two subgroups whose abundances oscillated synchronously (Fig. 6b).

\section{Discussion}

It is well known that unstable host-parasitoid interactions can be stabilized when species interact in a metapopulation structure (Hassell 2000, Bonsall et al. 2002, Briggs and 
(a)

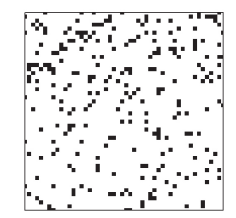

$$
\mathrm{pr}=0.6
$$

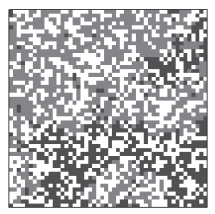

$\mathrm{pr}=0.2$
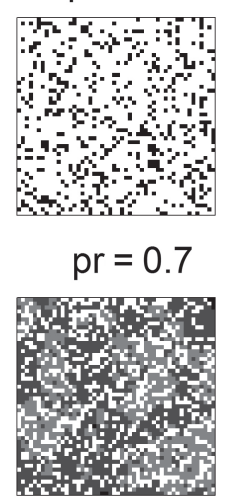
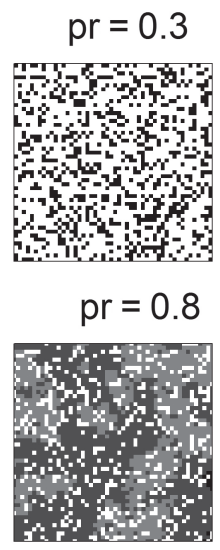

$\mathrm{pr}=0.3$

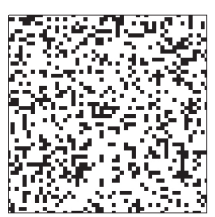

$\mathrm{pr}=0.8$

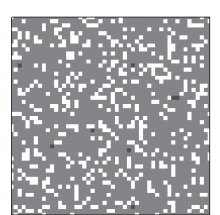

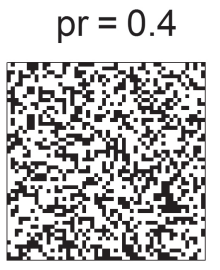

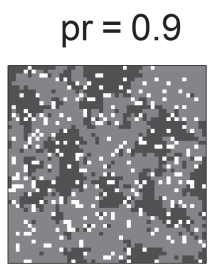

$$
\mathrm{pr}=0.4
$$

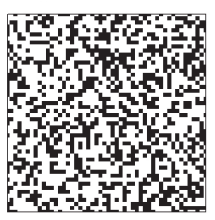

$\mathrm{pr}=0.9$

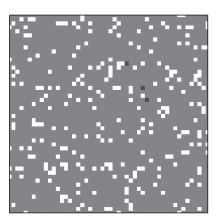

$\mathrm{pr}=0.5$

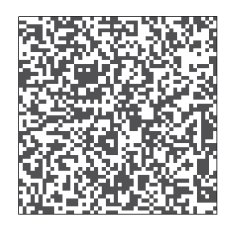

$\mathrm{pr}=1.0$

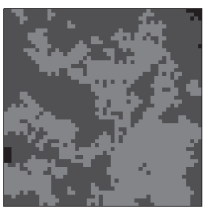

$\mathrm{pr}=0.5$

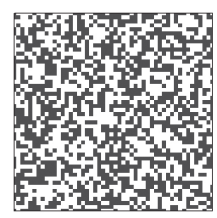

$\mathrm{pr}=1.0$
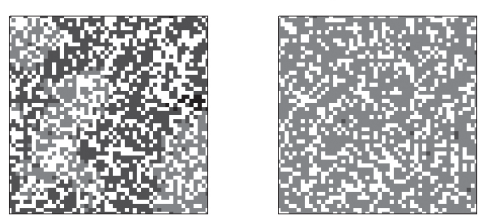

Figure 5. Plots showing the spatial occurrence of interacting species at different probabilities of resource occurrence after 10000 time-steps over a grid of $50 \times 50$ sites with reflective boundary conditions. Black represents the presence of only the basal ephemeral resource, dark gray represents the occurrence of the basal resource and hosts and light gray represents the occurrence of the basal resource, hosts and parasitoids at a site. (a) $k=1600$ and (b) $1600 \leq k_{\text {rand }} \leq 8000$.

Hopes 2004). In this study, we have shown that an extinction-prone host-parasitoid system, as observed empirically and theoretically with $C$. megacephala and $N$. vitripennis, can persist in metapopulations with ephemeral local patches (MELP). In addition, the empirical and theoretical results showed that demographic parameters and characteristics of basal ephemeral resources are essential for host-parasitoid coexistence in a MELP system.

As observed in experimental scenario 1, parasitoids tended to overexploit hosts and drive them to extinction. However, in scenario 2, with only two sites and one active patch at each generation, some replicates achieved host persistence and parasitoid extinction. This indicated that local demographic processes, such as host carrying capacity, interference competition and parasitoid control of sex ratio, influenced the species dispersal rates and, consequently, the colonization of new patches. This allowed the appearance of local patches with no parasitoids, pointing to a possible co-existence in larger systems. This behavior was confirmed in our model host-parasitoid simulation in the MELP $(k=1600)$.

Our experiment was designed to measure as many demographic parameters as possible within a simple setup. These results were then extrapolated to more realistic situations using simulations. Because the experimental data was highly variable, the estimation of the parameters used for the response functions of the model was subject to natural variation. By changing the dispersal rates $\mu_{F}$ and $\mu_{H}$ or the attack $a$, we obtained coexistence in smaller arenas in the simulations. This result indicates that the model is qualitatively robust and that coexistence is indeed a result of spatial structure. However, the specific details of how large the arena must be to achieve coexistence depend on the demographic parameters, which here were determined only approximately.

Our computer simulations also showed that fluctuations in population densities within a patch, acting concomitantly with the stochastic distribution of resources, directly affected the number of migrants and patch colonization, which resulted in patterns of distribution over the landscape.

Previous studies have shown that density fluctuations can increase the extinction risk of local populations, particularly when the populations are small and the patch is ephemeral (Lande 1993, Legendre et al. 1999). In this context, many studies have reported the negative effects of large carrying capacity on the stability of the metapopulation via the occurrence of extinction events (Lande 1993, Sæther et al. 1998). Bonsall et al. (2002) demonstrated that narrow density fluctuations can disrupt the regulation within local patches and can lead the metapopulation to extinction.

In our simulations with low carrying capacity, i.e. a poor-quality environment $(k=1600)$, the host density in 

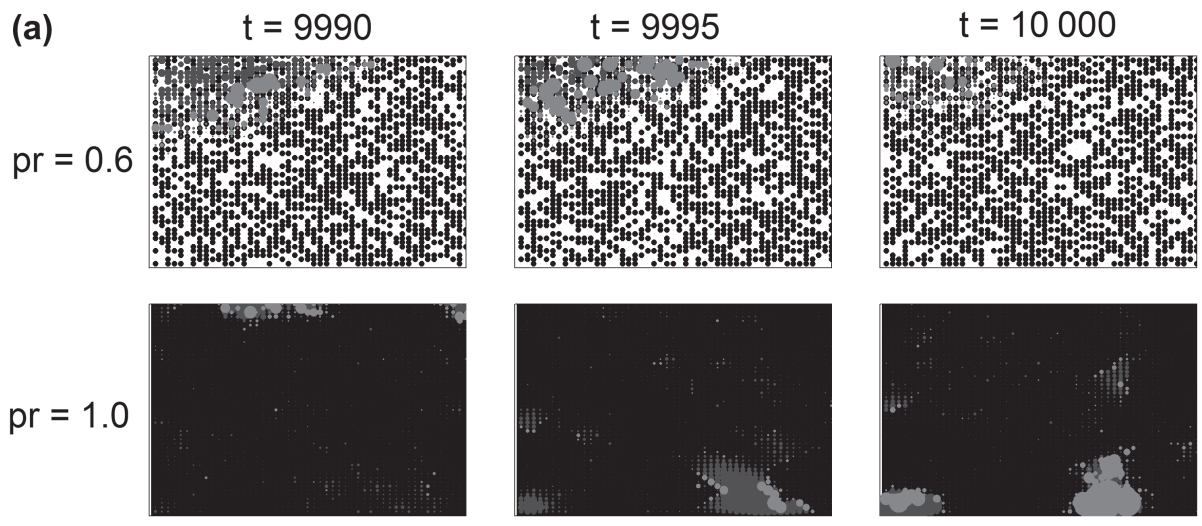

(b)

$t=9990$
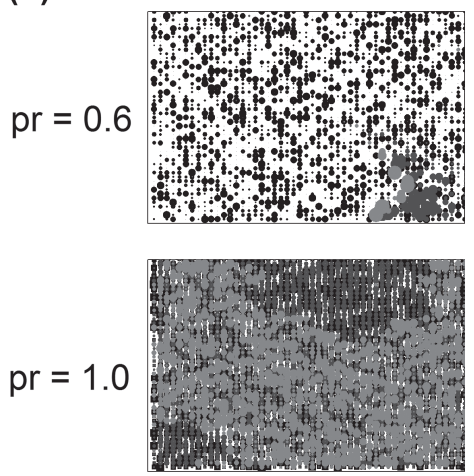

$t=9995$
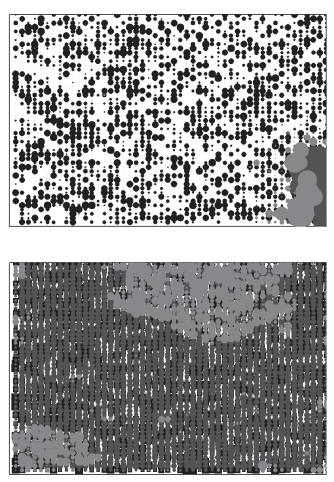

$t=10000$
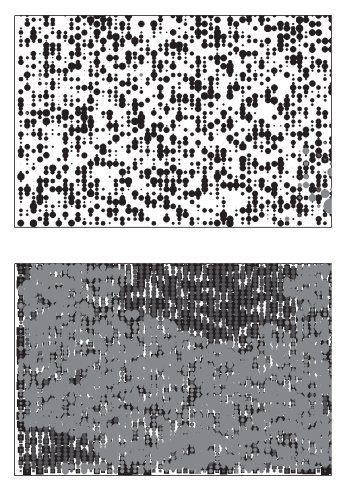

Figure 6. Snapshots of basal resource, host and parasitoid spatial occupancy for 9990, 9995 and 10000 time-steps over a grid of $50 \times 50$ sites with reflective boundary conditions. Black, dark gray and light gray symbols represent, respectively, the basal ephemeral resource, host and parasitoid abundances within each patch. The sizes of the dots are proportional to the population at each site. We compared the effects of two probabilities of basal resource site occupancy $(p r=0.6$ and $p r=1.0)$ for a spatial pattern of host and parasitoid abundance distribution. (a) $k=1600$ and (b) $1600 \leq k_{\text {rand }} \leq 8000$. Parameter values: $\lambda=1.5 ; \alpha=0.34 ; \beta=0.06 ; c=20 ; \gamma=0.3 ; \mu_{H}=0.85, \mu_{F}=0.4$.

the corresponding patches was low. This scenario favored an increase in the number of males in the parasitoid progeny and consequently a decrease in the parasitoid dispersers (females). Combined with the higher dispersal and spread rates of hosts as compared to parasitoids, the host-parasitoid dynamics in the MELP developed traveling wave patterns of species distribution.

In simulations with larger resource sizes, the fluctuations in population density were smaller, favoring the increase of dispersed parasitoids. The larger number of migrants allowed the parasitoids to spread to all susceptible patches in the landscape, changing the spatial pattern of species distribution in the MELP. In simulations with larger carrying capacities, the MELP displayed less local extinction, and parasitoids were present in all patches containing hosts.

Many studies have shown the influence of dispersal, landscape structure and local population synchrony in population persistence (Bascompte and Solé 1995, Hanski 1999, Hassell 2000). In contrast, little is known about the dynamics of a community that depends on ephemeral basal resources to persist. In the present study, the local persistence of parasitoids depends on the host metapopulation dynamics, which, in turn, depends mainly on the distribution basal ephemeral resources.

Previous studies (Price 1991, Hawkins 1992) have shown the importance of bottom-up effects to the parasitoid com- munity (third trophic level). The lower trophic levels (host resources) can constrain the number and strength of trophic links in host-parasitoid webs (Price 1991). We found that resource sizes and their spatial distribution are important factors for determining the number of host and parasitoid migrants. The variability in ephemeral resource sizes (fixed or random) affected the species' spatial distribution pattern and coexistence was possible only for high probabilities of ephemeral resource occurrences in the landscape.

Host-parasitoid persistence in the MELP was negatively affected by the dynamics within ephemeral patches, by the isolation of ephemeral subpopulations (due to low occurrence of resources in sites) and by low numbers of total resources. The results showed that host-parasitoid persistence requires a minimum number of patches in the landscape, which in turn depends upon the probability of resource allocation.

On the other hand, non-persistence of hosts and parasitoids can be related to the isolation of patches in the landscape. In the case of a sufficiently high density of patches, several clusters of host-parasitoid populations formed because only that part of the landscape was reached by subpopulations. These cluster areas were linked by dispersion and helped to ensure the persistence of both species.

Some studies that used mark-recapture data and/or monitoring of patch occupation have reported the importance of 
migration rates for habitat patch sizes and isolation (Hanski and Ovaskainen 2000, Ehrlich and Hanski 2004, Altermatt and Ebert 2010). Others have emphasized the importance of ephemeral subpopulations for promoting emigration and persistence of species in metapopulations (Crone et al. 2001). Data regarding the assessment of the origins and numbers of migrants in empirical studies are rare and few empirical studies have analyzed these factors (Hanski and Gaggiotti 2004). The results obtained from microcosm experimentation have shown that understanding the factors affecting species movement can involve the combination of many elements, such as foraging behavior, parent-offspring conflict, ecological processes, selection pressure and the evolutionary history of the species.

We demonstrated that the increase in the carrying capacity, larger patch sizes, lead to the increase in migrant numbers and consequently increased the contribution of the species to populations in new patches in the MELP spatial structure. When landscape resources were low, coexistence was not observed. The importance of demographic fluctuations and environmental factors for the density of migrants and patch connectivity for host-parasitoid persistence in the MELP were evident in this study.

In summary, we studied the dynamics of hosts and parasitoids on a spatial structure where resources were short lived and randomly assigned to sites at every generation. We showed that this ephemeral metapopulation can lead to persistence of both species depending on the number of sites with resources available in the arena, i.e., the number of patches. Although we focused on particular traits of the host-parasitoid interaction, our approach can be applied to other types of interactions with stochastic environmental characteristics (e.g. ephemeral resources and fragmented habitats) and demographic processes (e.g. arising from behavior, genetic and physiological factors). The results presented here can also be relevant to the control of patchily distributed pests, which can be maintained by the occurrence of ephemeral resources in the landscape.

Acknowledgements - We thank A. X. Linhares and R. J. da Silva for laboratory support assistance, S. B. L. Araújo for help during the elaboration of the mathematical model and D. L. Brancoli for technical assistance. The work was supported by Fundação de Amparo à Pesquisa do Estado de São Paulo - FAPESP (grants no. 2009/05743-6 and 2009/11032-5).

\section{References}

Altermatt, F. and Ebert, D. 2010. Populations in small, ephemeral habitat patches may drive dynamics in a Daphnia magna metapopulation. - Ecology 91: 2975-2982.

Amat, I. et al. 2009. Differential use of conspecific-derived information by sexual and asexual parasitic wasps exploiting partially depleted host patches. - Behav. Ecol. Sociobiol. 63: 563-572.

Bascompte, J. and Solé, R. V. 1995. Rethinking complexity: modeling spatiotemporal phenomena in ecology. - Trends Ecol. Evol. 10: 361-366.

Baumgartner, D. L. and Greenberg, B. 1984. The genus Chrysomya (Diptera: Calliphoridae) in the New World. - J. Med. Entomol. 21: 105-113.
Beddington, J. R. et al. 1975. Dynamic complexity in predator-prey models framed in difference equations. - Nature 255: 58-60.

Bonsall, M. B. et al. 2002. Metapopulation structures affect persistence of predator-prey interactions. - J. Anim. Ecol. 71: 1075-1084.

Briggs, C. J. and Hoopes, M. F. 2004. Stabilizing effects in spatial parasitoid-host and predator models: a review. - Theor. Popul. Biol. 65: 299-315.

Burton-Chellew, M. N. et al. 2008. Facultative sex ratio adjustment in natural populations of wasps: cues of local mate competition and the precision of adaptation. - Am. Nat. 172: 393-404.

Charnov, E. L. 1976. Optimal foraging: the marginal value theorem. - Theor. Popul. Biol. 9: 126-136.

Crawley, M. J. 2007. The R book. - Wiley.

Crone, E. E. et al. 2001. Ecological influences on the dynamics of a field vole metapopulation. - Ecology 82: 831-843.

Ehrlich, P. R. and Hanski, I. 2004. On the wings of checkerspots: a model system for population biology. - Oxford Univ. Press.

Fauvergue, X. et al. 2006. Habitat assessment by parasitoids: consequences for population distribution. - Behav. Ecol. 17: $522-531$.

French, D. and Travis, J. 2001. Density-dependent dispersal in host-parasitoid assemblages. - Oikos 95: 125-135.

Fretwell, S. D. and Lucas, H. L. 1970. On territorial behavior and other factors influencing habitat distribution in birds. - Acta Biotheor. 19: 16-36.

Godfray, H. C. J. 1994. Parasitoids: behavioral and evolutionary ecology. - Princeton Univ. Press.

Grassberger, M. and Frank, C. 2003. Temperature-related development of the parasitoid wasp Nasonia vitripennis as forensic indicator. - Med. Vet. Entomol. 17: 257-262.

Grillenberger, B. K. et al. 2009a. Reproductive strategies under multiparasitism in natural populations of the parasitoid wasp Nasonia (Hymenoptera). - J. Evol. Biol. 22: 460-470.

Grillenberger, B. K. et al. 2009b. Female dispersal and isolationby-distance of Nasonia vitripennis populations in a local mate competition context. - Entomol. Exp. Appl. 132: 147-154.

Hamilton, W. D. 1967. Extraordinary sex ratios. - Science 156: 477-488.

Hanski, I. 1999. Metapopulation ecology. - Oxford Univ. Press.

Hanski, I. and Ovaskainen, O. 2000. The metapopulation capacity of a fragmentedland scape. - Nature 404: 755-758.

Hanski, I. and Gaggiotti, O. E. 2004. Ecology, genetics and evolution of metapopulations. - Elsevier.

Harrison, S. 1991. Local extinction in a metapopulation context: an empirical evaluation. - Biol. J. Linn. Soc. 42: 73-88

Hassell, M. P. 1978. The dynamics of arthropod predator-prey systems. - Princeton Univ. Press.

Hassell, M. P. 2000. Host-parasitoid population dynamics. - J. Anim. Ecol. 69: 543-566.

Hassell, M. P. et al. 1991. Spatial structure and chaos in insect population dynamics. - Nature 353: 255-258.

Hawkins, B. A. 1992. Parasitoid-host food webs and donor control. - Oikos 65: 159-162.

Lande, R. 1993. Risks of population extinction from demographic and environmental stochasticity and random catastrophes. - Am. Nat. 142: 911-927.

Legendre, S. et al. 1999. Demographic stochasticity and social mating system in the process of extinction of small populations: the case of passerines introduced to New Zealand. - Am. Nat. 153: 449-463.

Levins, R. 1969. Some demographic and genetic consequences of environmental heterogeneity for biological control. - Bull. Entomol. Soc. Am. 15: 237-240.

Molbo, D. and Parker, E. D. J. 1996. Mating structure and sex ratio variation in a natural population of Nasonia vitripennis. - Proc. R. Soc. B 263: 1703-1709. 
Murphy, D. D. et al. 1990. An environment-metapopulation approach to population viability analysis for a threatened invertebrate. - Conserv. Biol. 4: 41-51.

Outreman, Y. et al. 2005. Effects of within- and amongpatch experiences on the patch-leaving decision rules in an insect parasitoid. - Behav. Ecol. Sociobiol. 58: 208-217.

Price, P. W. 1991. The plant vigor hypothesis and herbivore attack. - Oikos 62: 244-251.
Sæther, B. E. et al. 1998. Environmental stochasticity and extinction risk in a population of a small songbird, the great tit. - Am. Nat. 151: 441-450.

Shuker, D. M. et al. 2006. Male influence on sex allocation in the parasitoid wasp Nasonia vitripennis. - Behav. Ecol. Sociobiol. 59: 829-835.

Whiting, A. R. 1967. The biology of the parasitic wasp Moroniella vitripennis [ = Nasonia brevicornis] (Walker). - Q. Rev. Biol. 42: 333-406.

\section{Appendix 1}

\section{Carrying capacity of used resource size}

To quantify the carrying capacity $(k)$ of the resource used in the lab microcosm, we offered $200 \mathrm{~g}$ of ground beef to seven densities of hosts in the first larval stage: 200, 500, 1000, 1500, 2000, 2500 and 3000 hosts. After feeding, the host pupae (survival larvae) were recorded to obtain the carrying capacity of the resource size used in the experimental microcosm. The carrying capacity $(k)$ of $200 \mathrm{~g}$ of basal resource was quantified around 1600 host individuals (Fig. A1.1).

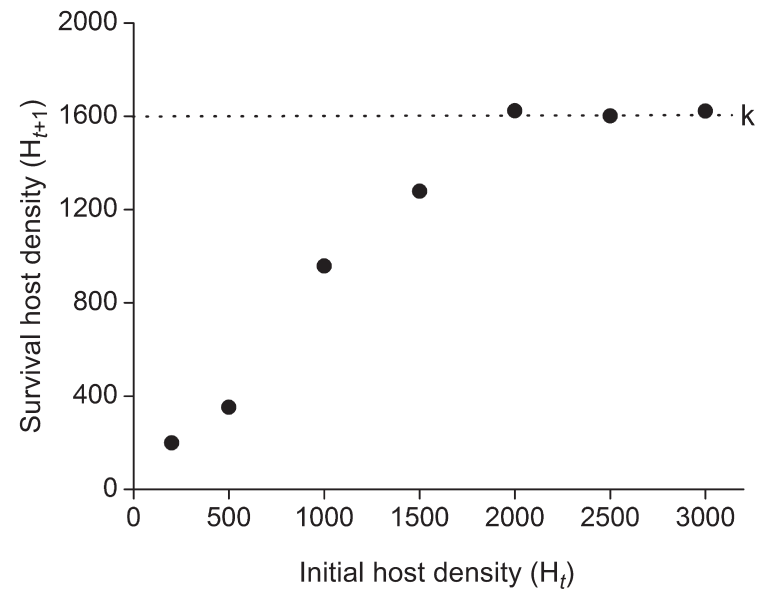

Figure A1.1. Experimental carrying capacity used in the interaction arenas for scenario 1 and scenario 2. 


\section{Appendix 2}

\section{Demographic parameter functions}

\subsection{Functional response}

To find a function to describe the functional response of female parasitoids $(p(H, N))$ in the absence and presence of conspecific competitors, we performed two experiments. First, we offered seven densities of hosts $(1,15,25,35,50,70$ and 100) to single $N$. vitripennis females. Second, we offered 10 host pupae to six densities of parasitoids $(1,5,10,15,20$ and 25) to obtain the proportion of parasitized hosts for each parasitoid foundress density. The parasitized hosts were recorded to obtain the functional response of $N$. vitripennis. These experimental sets were performed in vials $(20 \mathrm{~cm}$ height $\times 15 \mathrm{~cm}$ diameter $)$ maintained
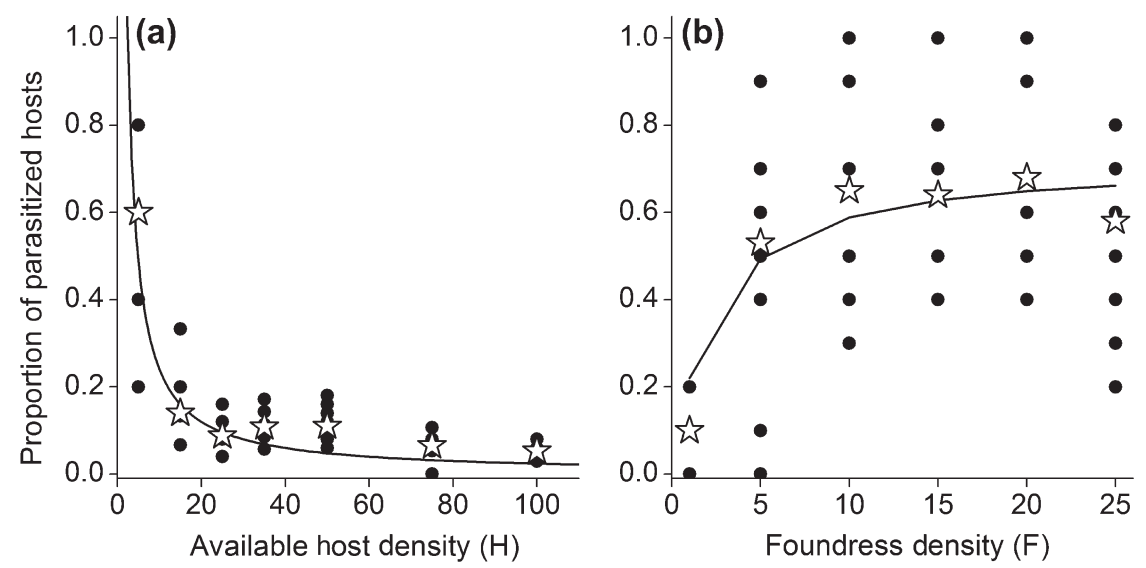

Figure A2.1. Parasitism of $N$. vitripennis on $C$. megacephala pupae in two cases: (a) one female parasitoid interacting with densities of host pupae and (b) densities of $N$. vitripennis interacting with 10 C. megacephala pupae. Note that, in the first case, the parasitism is not influenced by interference competition; in the second case, the parasitism is given with conspecific competition effects. Dots represent the experimental data, stars represent the averages obtained from experimental data and solid lines represent the functional responses of $N$. vitripennis given by the function $p(H, F)$ in Eq. 2. (a) $\mathrm{n}=70, \mathrm{DF}=69 ; \alpha=1.2( \pm 0.07) ; \beta=0 ; \mathrm{p}<0.001$ and (b) $\mathrm{n}=60 ; \mathrm{DF}=58$; $\alpha=0.34( \pm 0.05) ; \beta=0.06( \pm 0.01) ; \mathrm{p}<0.001$.

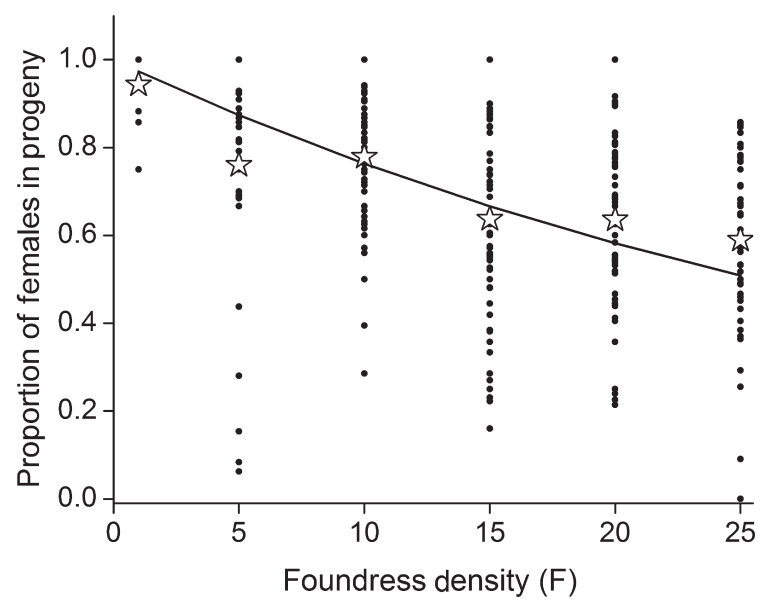

Figure A2.2. Proportion of females in N. vitripennis progeny for different foundress densities in patches. Dots represent the experimental data, stars represent the averages obtained from the experimental data and solid lines represent the sex ratio changes in $N$. vitripennis progeny given by the function $s(H, F)$ in Eq. 3. $\mathrm{n}=310 ; \mathrm{DF}=309 ; \gamma=0.3( \pm 0.016) ; \mathrm{p}<0.001$. 


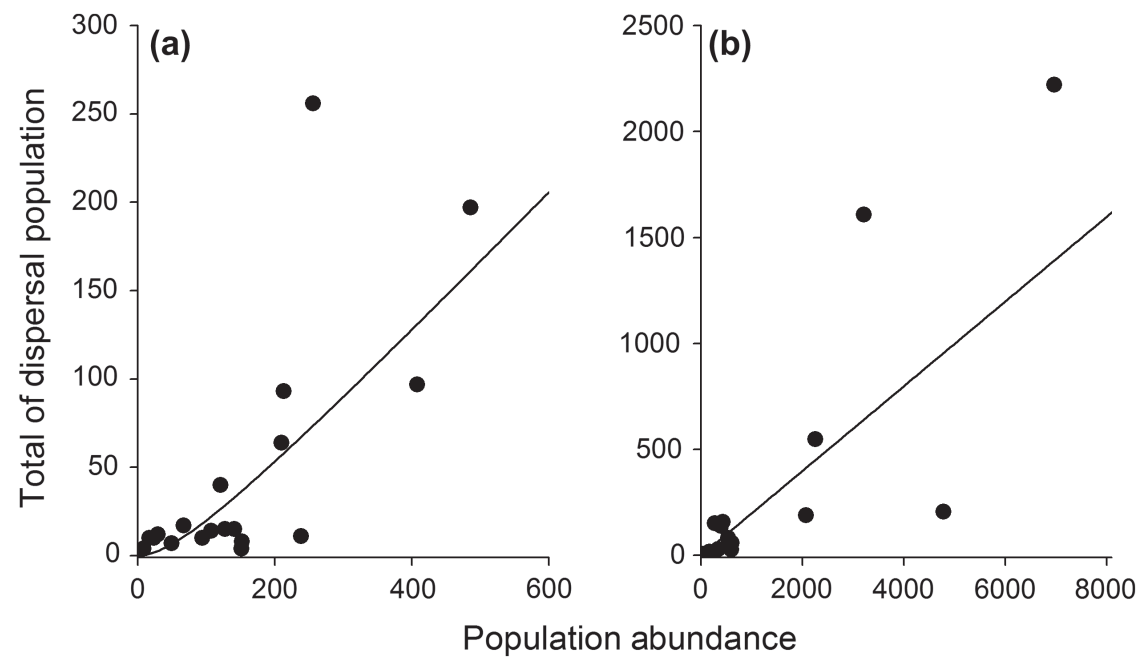

Figure A2.3. Variation of disperser averages in (a) host and (b) parasitoid populations. Dots represent the experimental data, and solid lines represent the dispersal population changes given by the theoretical model (Eq. 4 and 7, respectively). For host dispersion: $\mathrm{n}=19 ; \mathrm{DF}=18$; $\mu_{H}=0.85$ ( \pm 0.08$) ; \mathrm{p}<0.001$. For parasitoid dispersion: $\mathrm{n}=15, \mathrm{DF}=14 ; \mu_{F}=0.4( \pm 0.05) ; \mathrm{p}<0.001$.

in previously described laboratory-controlled conditions. For each set, we performed 10 replicates. (Fig. A2.1).

\subsection{Sex ratio adjustment}

The parasitized pupae obtained from the second functional response experiment were individually placed into gelatin capsules, and the number of female and male parasitoids was recorded for each parasitoid foundress density to obtain the function for the $N$. vitripennis sex ratio adjustment $s(H$, $E)$ ). In this case, for each foundress density, we considered all parasitized pupae found in vials as replicates (we used $\mathrm{n}=310$ ) (Fig. A2.2).

\subsection{Host and parasitoid dispersal rates}

To define the dispersal rates, we used the data obtained from scenario 2 . The numbers of hosts and female parasitoids that dispersed to the new patch and the numbers of individuals that remained at the native site were recorded to define the dispersal functions (respectively, $b^{\text {out }}$ and fout) (Fig. A2.3). 